University of Wollongong

Research Online

Faculty of Engineering - Papers (Archive)

Faculty of Engineering and Information

Sciences

$1-1-2006$

\title{
Direct kinematics and analytical solution to 3RRR parallel planar
} mechanisms

Denny Oetomo

Monash University

Hwee Choo Liaw

Monash University

Gursel Alici

University of Wollongong, gursel@uow.edu.au

Bijan Shirinzadeh

Monash University

Follow this and additional works at: https://ro.uow.edu.au/engpapers

Digitedrt of the Engineering Commons

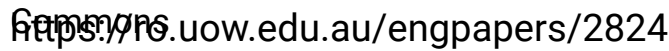

Network

Logo

Recommended Citation

Oetomo, Denny; Liaw, Hwee Choo; Alici, Gursel; and Shirinzadeh, Bijan: Direct kinematics and analytical solution to 3RRR parallel planar mechanisms 2006, 2251-2256.

https://ro.uow.edu.au/engpapers/2824

Research Online is the open access institutional repository for the University of Wollongong. For further information contact the UOW Library: research-pubs@uow.edu.au 


\title{
Direct Kinematics and Analytical Solution to 3RRR Parallel Planar Mechanisms
}

\author{
Denny Oetomo*, Hwee Choo Liaw*, Gursel Alici ${ }^{\dagger}$, Bijan Shirinzadeh* \\ *Department of Mechanical Engineering \\ Monash University, Clayton, Victoria 3800 Australia \\ Email: \{denny.oetomo, hwee-choo.liaw, bijan.shirinzadeh\}@eng.monash.edu.au \\ ${ }^{\dagger}$ School of Mechanical, Materials, and Mechatronics Engineering \\ University of Wollongong, NSW 2522, Australia. \\ Email: gursel@uow.edu.au
}

\begin{abstract}
This paper presents the direct kinematic solutions to 3DOF planar parallel mechanisms. Efforts to solve the direct kinematics of planar parallel mechanisms have concentrated on RPR mechanisms due to its inherent simplicity. It is established that the direct kinematic equations of a general 3DOF planar parallel mechanism can be reduced to a univariate polynomial of degree 8. This paper presents the derivation of this univariate polynomials for both 3RRR and 3RPR mechanisms, showing the similarities and differences between the two common configurations of 3DOF planar parallel mechanisms. This paper also presents the on the direct kinematic solution to a simplified case of the 3RRR planar parallel mechanisms, where it is possible to decouple the polynomial further into two quadratic equations, describing the position and orientation of the end-effector, respectively. This result will provide an efficient computation method for a very useful configuration of planar parallel manipulators.
\end{abstract}

Keywords—direct kinematics, analytical solution, planar parallel mechanism, 3RRR

\section{INTRODUCTION}

Parallel (or closed-chained) mechanisms play an important part in the design of mechanisms requiring rigidity of structure and precise motion control. These advantages come at the expense of the workspace of the mechanisms, which is considerably smaller than that of a serial manipulator. It can be loosely described that the workspace of a parallel mechanism is the intersection of the workspace of the individual 'serial linkages' connecting the moving platform of the mechanism to its base. When a parallel mechanism is capable of meeting the workspace requirement of a task, it is often the preferred option due to its structural rigidity. This would also result in less disturbances from mechanical vibration and better accuracy in motion control.

It is well-known that the forward kinematic solutions of a parallel mechanism is difficult to obtain. This is because the independent equations describing the geometric relationship between different chains are related to one another in nonlinear manners. Although closed-form solutions are often desired or even preferred, the solution is often complex. Many solutions proposed in the past have concentrated on numerical methods, such as Newton-Raphson [1][2], and learning networks [3].
Conventional numerical methods is not only computationally expensive when implemented to calculate the direct kinematics of a parallel manipulator online, but it also does not guarantee to yield all the possible solutions, hence there is a possibility of missing a critical solution. Didrit et. al [4] refined the numerical methods using interval analysis and provided all possible solutions to a parallel mechanism without prior knowledge of the number of solutions and initial estimate for the iterative scheme.

It is also well established that the direct kinematics problem of parallel mechanisms can present a prohibitively large number of solutions, as presented in [5], where StewartGough platform was reported to admit 40 real solutions. The real-time computation of the roots of the polynomial is prohibitively expensive for online implementation. There is also an issue in identifying the actual solution in the control process. To simplify the problem, different approaches have been proposed, such as through special configurations [6][7] and extra sensors [8][9]. Gosselin and Merlet [6] presented simplified closed-form solutions for planar parallel mechanism by introducing collinearity between the revolute joints of the base and the platform of the mechanism. This is further refined in [10], where three types of simplifications were formalised for a 3DOF planar parallel manipulator with RPR (revoluteprismatic-revolute) linkages. Closed form solutions were also investigated with additional sensors which reduce the complexity of the nonlinear equations [8][9]. Solution of a Stewart Platform mechanism was also presented in [11]. The solution resulted in decoupled polynomial equations of overall degree of sixteen and no iterative scheme were required. In avoiding the iterative process, some of the solutions to the polynomials were obtained algebraically, while others through geometrical reasoning. This method does not cover the most general cases of configurations, although it provides very useful insight into the practical closed-form solutions of the problem.

In this paper, the two most common configurations of 3DOF planar parallel mechanisms, namely the 3RRR and the 3RPR configurations, are studied. These mechanisms form one of the three categories of 3DOF planar parallel mechanisms as presented in [12]. Since the 3DOF planar parallel mechanism 
is made up of three chains connecting the base to the moving platform, each describing a circular locus with a quadratic equation, the resulting polynomial solution has a natural order of $2^{3}=8$, admitting a maximum of 6 real solutions [13]. Minimal solutions were presented for specific cases of simplified 3DOF planar parallel mechanisms in [6] and more recently in [10] and [14]. These minimal solutions in the form of sixth degree univariate polynomials were further formulated for specific examples of 3DOF planar parallel mechanisms to reduce the complexity of the solutions to polynomials of order 4 or less, making it possible for closed-form solutions to be calculated. These simplified mechanisms are termed analytic 3DOF planar parallel mechanisms, as close-form analytic solutions exist for their direct kinematic problems. In all these papers, however, efforts were concentrated on 3RPR (Revolute-Prismatic-Revolute) planar mechanisms.

In this paper, we present the derivation of the direct kinematics of both the 3RRR and the 3RPR mechanisms. Although it has been claimed that the direct kinematics of this configuration (3RRR) can be treated in the same way as that of 3RPR's, it has never been presented explicitly. It also provides a misleading understanding that $3 R R R$ planar parallel mechanisms can be solved in a similar manner to obtain the closed-form solutions. This is often not the case, because each RRR link comprises of two cascaded circular arcs of constant radius, compared to the locus of one circular arc with stationary centres, albeit with variable radius, for an RPR link. In this paper, however, one special (simplified) 3RRR configuration is presented and its closed-form direct kinematic solution solved. This simplification decouples the solutions into two quadratic equations, essentially forming a $4^{\text {th }}$ degree polynomial.

It is also important to note that in a hardware control implementation where all computations have to be performed within a specified servo period, numerical iterative methods run the risk of exceeding the allowable computation time, if the residual error does not converge below a desired threshold. This could either cause inconsistency in calculation time or accumulated residual error in the direct-kinematic calculation. Compared to numerical iterative method, the existence of a closed-form solution provides a more accurate strategy in motion and force control of manipulators built with such designs, as the solution is exact and computationally efficient. The results in this paper are also important because 3RRR is a common and useful configurations for planar manipulation and positioning systems.

\section{Polynomial Solution to Direct Kinematics of the Planar Parallel Mechanisms}

A parallel mechanism is represented by the base of the mechanism and the moving platform where the end-effector is located. The base and the platform are connected by a series of linkages. The number of chains connecting the base and the platform of the mechanism is defined as $N$. For a planar 3DOF parallel mechanism, it is necessary to have a minimum of 3 chains $(N=3)$, each of the chain is capable of $2 \mathrm{DOF}$



Fig. 1. A general case 3RRR planar parallel mechanism

positioning in the plane of motion. A planar 3DOF parallel mechanism can have different configurations for each of its chain, however, for ease of design and fabrication, as well as to preserve the symmetry and to optimise the isotropy of the mechanism, it is often designed to have the same configuration for all of its chains. The list of all possible configurations for a 3DOF planar parallel mechanism is given in [12][15]. Out of the 21 possible configurations, it was shown that only 10 configurations were capable of producing 3DOF planar parallel manipulation [15], and they are represented by three major categories [12]. However, the most common configurations in use are the 3RPR (Revolute-Prismatic-Revolute) and 3RRR (Revolute-Revolute-Revolute), where the actuated joints are underlined.

We will start with a type 1 mechanism as defined in [12] as shown in Fig. 1. $B_{i}$, and $C_{i}$ and the task space variables $(x, y, \theta)$ are as defined in Fig. 2.

Three independent equations can be derived to describe the three circular loci of points $C_{i}$ forming the three independent closed-chains:

$$
\begin{gathered}
\left(x-b_{1 x}\right)^{2}+\left(y-b_{1 y}\right)^{2}=l_{1}^{2} ; \\
\left(x+d_{1} C_{\theta}-b_{2 x}\right)^{2}+\left(y+d_{1} S_{\theta}-b_{2 y}\right)=l_{2}^{2} ; \\
\left(x+d_{2} C_{\theta+\phi}-b_{3 x}\right)^{2}+\left(y+d_{2} S_{\theta+\phi}-b_{3 y}\right)=l_{3}^{2} ;
\end{gathered}
$$

where $S_{\theta}=\operatorname{Sin}(\theta), C_{\theta}=\operatorname{Cos}(\theta)$.

Equations (1)-(3) describe the circular loci of points $C_{1}$, $C_{2}, C_{3}$ with centres on $B_{1}, B_{2}, B_{3}$. Subtracting (1) from (2) and (3) reduces the system to the following set of equations: 


$$
\begin{aligned}
& \left(x-b_{1 x}\right)^{2}+\left(y-b_{1 y}\right)^{2}=l_{1}^{2} \\
& G_{1} x+G_{2} y+G_{3}=l_{2}{ }^{2}-l_{1}{ }^{2} ; \\
& G_{4} x+G_{5} y+G_{6}=l_{3}{ }^{2}-l_{1}{ }^{2}
\end{aligned}
$$

where

$$
\begin{gathered}
G_{1}=2 b_{1 x}-2 b_{2 x}+2 d_{1} C_{\theta} \\
G_{2}=2 b_{1 y}-2 b_{2 y}+2 d_{1} S_{\theta} \\
G_{3}=\left(b_{2 x}^{2}+b_{2 y}^{2}\right)-\left(b_{1 x}^{2}+b_{1 y}^{2}\right)+d_{1}^{2}-2 d_{1}\left(b_{2 x} C_{\theta}+b_{2 y} S_{\theta}\right) \\
G_{4}=2 b_{1 x}-2 b_{3 x}+2 d_{2} C_{\phi+\theta} \\
G_{5}=2 b_{1 y}-2 b_{3 y}+2 d_{2} S_{\phi+\theta} \\
G_{6}=\left(b_{3 x}^{2}+b_{3 y}^{2}\right)-\left(b_{1 x}^{2}+b_{1 y}^{2}\right)+d_{2}^{2}-2 d_{2}\left(b_{3 x} C_{\phi+\theta}+b_{3 y} S_{\phi+\theta}\right)
\end{gathered}
$$

Equations (5)-(6) can be solved simultaneously to yield the expressions for $(x, y)$ as a function of $\theta$ :

$$
\left[\begin{array}{l}
x \\
y
\end{array}\right]=\left[\begin{array}{ll}
G_{1} & G_{2} \\
G_{4} & G_{5}
\end{array}\right]^{-1}\left[\begin{array}{l}
l_{2}{ }^{2}-l_{1}{ }^{2}-G_{3} \\
l_{3}{ }^{2}-l_{1}{ }^{2}-G_{6}
\end{array}\right]
$$

These are substituted into (4) to produce a univariate equation in terms of the trigonometric functions of $\theta$. The Tangent Half-Angle formula is then utilised by substituting:

$$
\sin (\theta)=\frac{2 T}{\left(1+T^{2}\right)}
$$

and

$$
\cos (\theta)=\frac{\left(1-T^{2}\right)}{\left(1+T^{2}\right)}
$$

into the equation to obtain a univariate polynomial degree 8 :

$$
\sum_{i=0}^{8} a_{i} T^{i}=0
$$

where

$$
T=\tan \left(\frac{\theta}{2}\right)
$$

The solution to this polynomial yield the solutions to angle $\theta$, which are then substituted into (13) to obtain the solutions to the task space position $(x, y)$.

\section{A. $3 R \underline{P R}$}

For a 3RPR planar parallel mechanism with actuated prismatic joints, the polynomial solution to the direct kinematics problem is obtained by setting $\left[b_{i x}, b_{i y}\right]^{T}$, where $i=1,2,3$ as constant (stationary points). It should be noted that $\left[l_{1}, l_{2}, l_{3}\right]^{T}$ are the actuated joints / variables and their values, representing the actuator displacement, can be measured and are therefore known. If the origin of the operational frame is defined at point $B_{1}$, and the x-axis of the frame is defined to go through point $B_{2}$, then the equations can be simplified by setting $\left[b_{1 x}, b_{1 y}\right]^{T}=[0,0]^{T}$, and $b_{2 y}=0$.

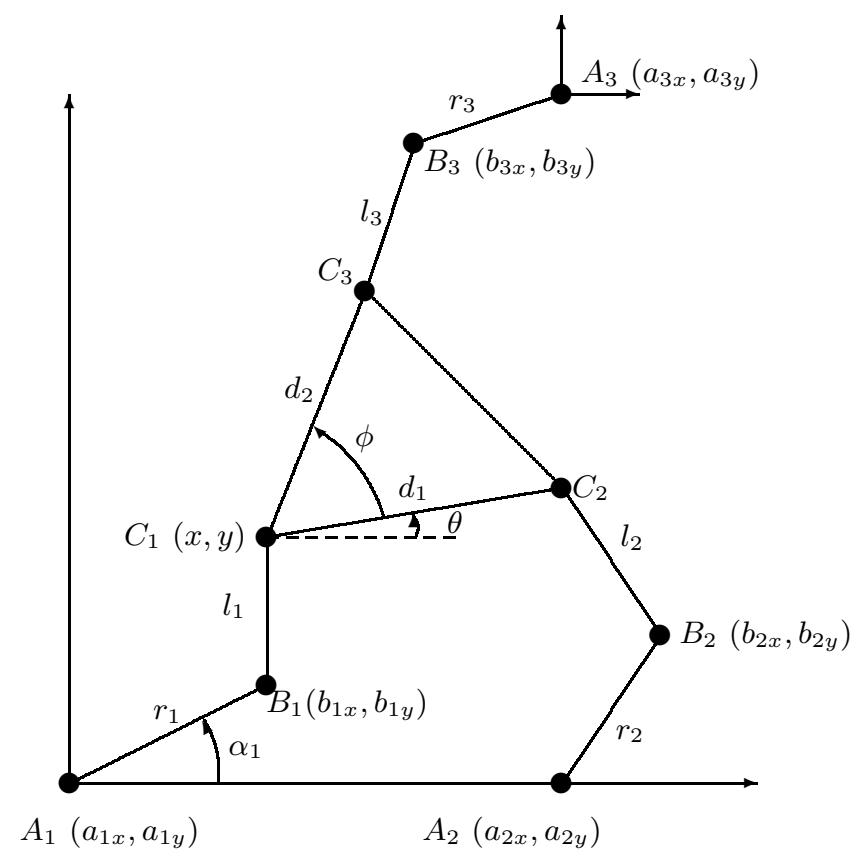

Fig. 2. A general case 3RRR planar parallel mechanism

\section{B. $3 R R R$}

For a 3RRR planar parallel mechanism with actuation on the first joint of the RRR link, the polynomial solution to the direct kinematics problem is obtained by setting $\left[b_{i x}, b_{i y}\right]^{T}=$ $\left[r_{i} C_{\alpha_{i}}, r_{i} S_{\alpha_{i}}\right]^{T}$ (see Fig. 2). The actuated joints / variables in this case would be angles $\left.\alpha_{1}, \alpha_{2}, \alpha_{3}\right]^{T}$.

\section{Closed Form Solutions for 3DOF PlanaR Parallel Mechanisms}

Currently, three types of analytic 3DOF planar parallel mechanisms have been identified and formalised in [10]: 1 . mechanisms where two of the joints on the base or on the moving platform coincide, 2. mechanisms where joints $A_{1}$, $A_{2}$, and $A_{3}$ are aligned and $B_{1}, B_{2}$, and $B_{3}$ are aligned, 3 . mechanisms with similar platforms where the base and moving platforms are similar triangles.

All three types have been analysed for 3RPR mechanisms. It is also stated the mathematical derivation for both 3RRR and 3RPR mechanisms are the same. While this is true, it should be clarified that the special architectures leading to analytical expression for 3RPR mechanisms do not necessarily apply to 3RRR mechanisms. Examples of this work include [6], [10] and [14].

Sections II-A and II-B highlight the difference in the mathematical derivations of 3RPR and 3RRR mechanisms, respectively. It was noted that the special configurations that leads to the reduction of the degrees in the polynomial solutions of the direct kinematics problem were based on the assumptions of stationary points $B_{i}$. Hence, many of the simplifications (hence, special architectures) do not apply to 


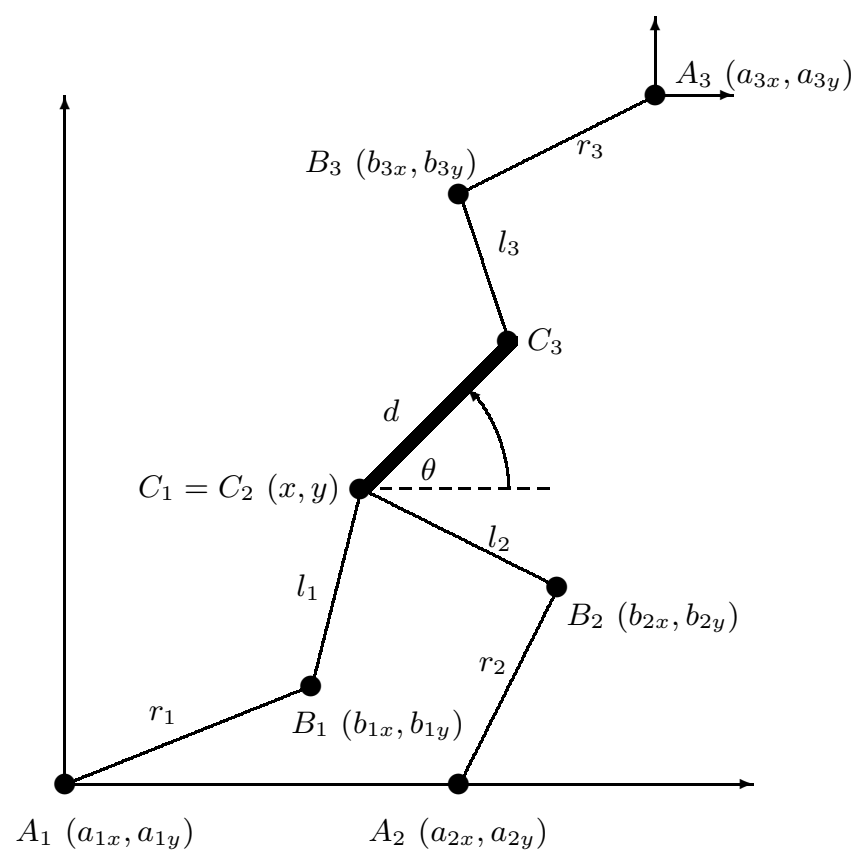

Fig. 3. Type I of the analytic planar parallel mechanism

3RRR mechanisms, as the position of points $B_{i}$ are dependent on the displacements of the actuated joints.

In this paper, we present a special architecture that apply to the 3RRR mechanism. This is equivalent to the first method of simplification as reported in [10], which is where points $C_{1}$ and $C_{2}$ (as defined in Fig. 2) are coincident.

\section{Closed-Form Solution to A Sub-Class of 3RRR PlanAR PARALLEL MECHANISMS}

The sub-class of the 3RRR analytic planar parallel mechanisms studied in this paper is where two of its three chains connecting the base and moving platforms have a common joint at one of their ends. This special sub-class provides simplification of the direct kinematic problem of the mechanism into two decoupled quadratic polynomials, essentially yielding four solutions but simpler to solve than a fourth degree polynomial.

In Fig. 3, a 3RRR analytic planar parallel mechanism is shown where joints $C_{1}$ and $C_{2}$ are coincident. As $d_{1}=0$, the notation can be simplified with $d$ representing the distance between the coincident joint $C_{1}=C_{2}$ and $C_{3}$. Orientation angle $\theta$ is defined as in Fig. 3 and constant variable $\phi$ is no longer required.

In a general 3DOF planar parallel mechanism, an independent closed-chain formed by the moving platform with two RPR chains essentially forms a four-bar mechanism, while a closed-chain of the moving platform with two RRR chains form a six-bar mechanism and hence is more complex to solve. However, defining $C_{1}$ and $C_{2}$ as coincident reduces the closed-chain to a five-bar mechanism and decouples the direct

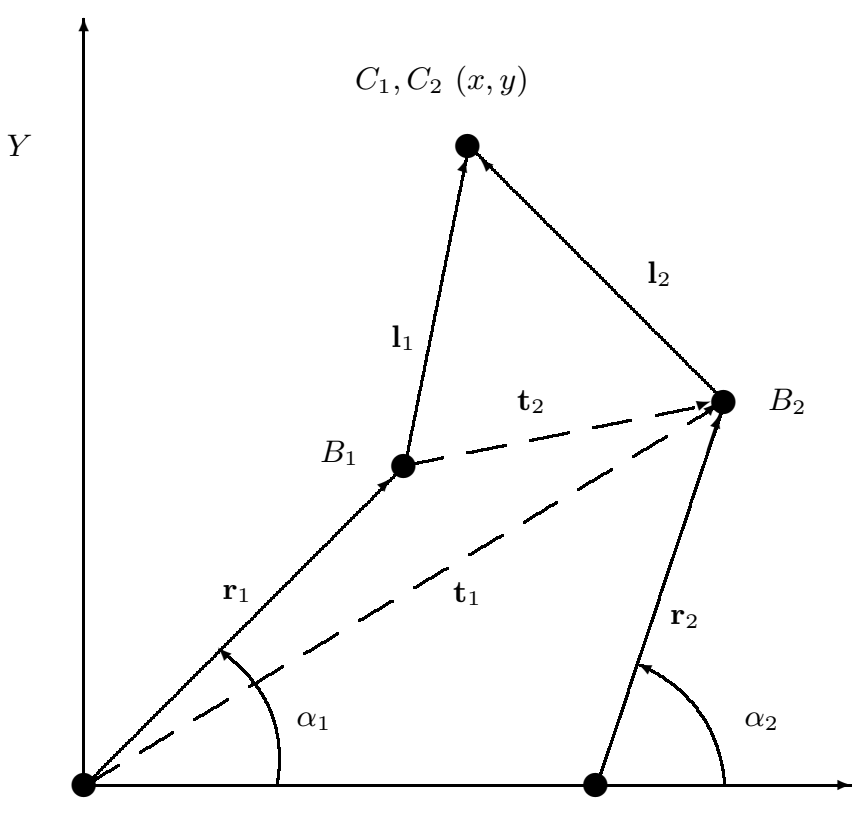

$A_{1}(0,0)$

$A_{2}\left(a_{2 x}, 0\right) \quad X$

Fig. 4. Treating the closed-chain $A_{1} B_{1} C_{1} B_{2} A_{2} A_{1}$ as a five bar mechanism

kinematics for $(x, y)$ from the solution of $\theta$. The following subsections will solve for $(x, y)$ and for $\theta$, respectively.

A. Analytic Solution for $(x, y)$

To solve for $(x, y)$, we treat the closed-chain $A_{1} B_{1} C_{1} B_{2} A_{2} A_{1}$ as a five-bar mechanism (Fig. 4). For the provided joint displacement inputs $\alpha_{1}$ and $\alpha_{2}$, and the specified link lengths, the analytical expressions for the coordinates of the output point $(x, y)$ can be obtained. The coordinates of points $B_{1}$ and $B_{2}$ which are the $\mathrm{x}$ and $\mathrm{y}$ components of vectors $\mathbf{r}_{1}$ and $\mathbf{t}_{1}$ can be considered as the coordinates of the centers of two circles of radii $l_{1}$ and $l_{2}$. Note that the centers of the two circles are expressed as functions of the inputs provided by the actuators fixed to the ground. It is well-established that the intersection of the two circles gives a maximum of two solutions which are the possible locations of $(x, y)$. An in-depth discussion of the kinematics and dynamics of a general five-bar mechanisms can be found in [16][17]. Referring to Fig. 5, the analytical expressions for these two solutions are obtained using the following algorithm:

$$
\begin{aligned}
& \mathbf{r}_{1}=r_{1} C_{\alpha_{1}} \mathbf{i}+r_{1} S_{\alpha_{1}} \mathbf{j}, \\
& \mathbf{t}_{1}=\left(a_{2 x}+r_{2} C_{\alpha_{2}}\right) \mathbf{i}+r_{2} S_{\alpha_{2}} \mathbf{j}, \\
& \mathbf{t}_{2}=\mathbf{t}_{1}-\mathbf{r}_{1}=D \mathbf{i}+E \mathbf{j}, \\
& D=a_{2 x}+r_{2} C_{\alpha_{2}}-r_{1} C_{\alpha_{1}} \text {, } \\
& E=r_{2} S_{\alpha_{2}}-r_{1} S_{\alpha_{1}} \text {, } \\
& t_{2}=\sqrt{D^{2}+E^{2}},
\end{aligned}
$$




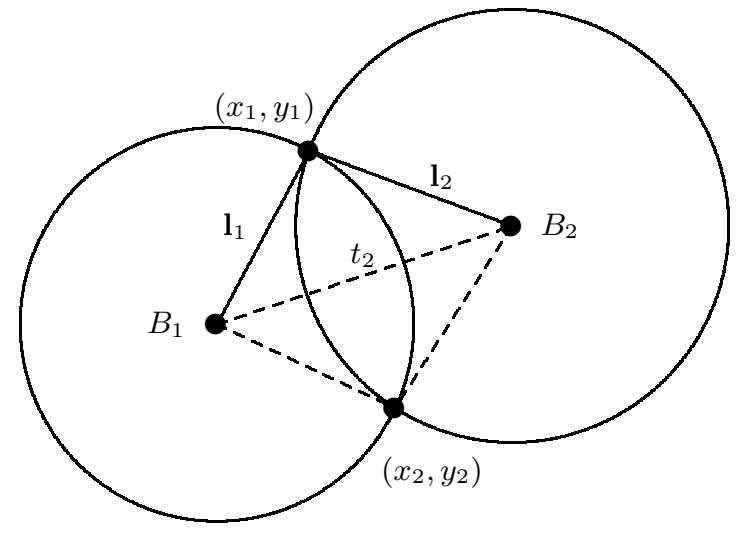

Fig. 5. The two solutions of the $A_{1} B_{1} C_{1} B_{2} A_{2} A_{1}$ five bar mechanism

$$
\begin{gathered}
F=\frac{l_{1}^{2}+t_{2}^{2}-l_{2}^{2}}{2 t_{2}^{2}}, \\
H=\sqrt{\frac{l_{1}^{2}}{t_{2}^{2}}-F^{2}} .
\end{gathered}
$$

The coordinates of $(x, y)$ have two possible solutions of $\left(x_{1}, y_{1}\right)$ and $\left(x_{2}, y_{2}\right)$ where:

$$
\begin{aligned}
& x_{1}=b_{1 x}+F D-E H, \\
& y_{1}=b_{1 y}+F E+D H, \\
& x_{2}=b_{1 x}+F D+E H, \\
& y_{2}=b_{1} y+F E-D H .
\end{aligned}
$$

\section{B. Analytic Solution for Orientation Angle $\theta$}

To solve for the orientation of the moving platform with respect to the base $(\theta)$, it can be derived that:

$$
\left(x+d C_{\theta}-b_{3 x}\right)^{2}+\left(y+d S_{\theta}-b_{3 y}\right)^{2}={l_{3}}^{2} .
$$

This essentially describes a circle of radius $l_{3}$ centred around $\left(b_{3 x}, b_{3 y}\right)$. As $(x, y)$ can be obtained independently of $\theta$ as shown in Section IV-A, they can be treated as known in (28).

Substituting (14) and (15) for the trigonometric functions, (28) can be rearranged into a quadratic equation of $T$,

$$
K T^{2}+L T+M=0
$$

where:

$$
\begin{gathered}
K=\left(b_{3 x}+d-x\right)^{2}+\left(b_{3 y}-y\right)^{2}-l_{3}{ }^{2}, \\
L=4 d\left(y-b_{3 y}\right), \\
M=\left(-b_{3 x}+d+x\right)^{2}+\left(b_{3 y}-y\right)^{2}-{l_{3}}^{2} .
\end{gathered}
$$

The two roots of the quadratic equations in $T$ yields two solutions to $\theta$ by:

$$
\theta=\tan ^{-1}\left(\frac{\sin (\theta)}{\cos (\theta)}\right)
$$

where $\sin (\theta)$ and $\cos (\theta)$ are obtained through (14) and (15).

\section{NumERICAL EXAMPLE AND VERIFICATION}

In this example, the following values are defined for the 3RRR planar parallel manipulator as shown in Fig. 3:

$$
\begin{aligned}
r_{1} & =r_{2}=r_{3}=0.4, \\
l_{1} & =l_{2}=l_{3}=0.3, \\
a_{2 x} & =0.6 \\
a_{3 x} & =1.0541 \\
a_{3 y} & =1.0454 \\
d & =0.3 .
\end{aligned}
$$

The end-effector pose is defined at $(x, y, \theta)^{T}=$ $(0.5,0.4, \pi / 6)^{T}$. All length units are in $m$.

Through inverse kinematics, it is obtained that:

$$
C_{\beta_{i}}=\frac{c_{i x}^{2}+c_{i y}^{2}-\left(l_{i}{ }^{2}+r_{i}{ }^{2}\right)}{2 l_{i} r_{i}}
$$

and

$$
\begin{gathered}
C_{\alpha_{i}}=\frac{\left(c_{i x}-a_{i x}\right)\left(r_{i}+l_{i} C_{\beta_{i}}\right)+\left(c_{i y}-a i y\right)\left(l_{i} S_{\beta_{i}}\right)}{\left(r_{i}+l_{i} C_{\beta_{i}}\right)^{2}+\left(l_{i} S_{\beta_{i}}\right)^{2}} \\
S_{\alpha_{i}}=\frac{\left(c_{i y}-a_{i y}\right)\left(r_{i}+l_{i} C_{\beta_{i}}\right)+\left(c_{i x}-a_{i x}\right)\left(l_{i} S_{\beta_{i}}\right)}{\left(r_{i}+l_{i} C_{\beta_{i}}\right)^{2}+\left(l_{i} S_{\beta_{i}}\right)^{2}} \\
\alpha_{i}=\tan ^{-1}\left(\frac{S_{\alpha_{i}}}{C_{\alpha_{i}}}\right)
\end{gathered}
$$

where $\left(c_{i x}, c_{i y}\right)$ are the $\mathrm{x}$ and y coordinates of points $C_{i}$. From Fig. 4, it is clear that

$$
\left(c_{1 x}, c_{1 y}\right)^{T}=\left(c_{2 x}, c_{2 y}\right)^{T}=(x, y)^{T}
$$

and that

$$
\left[\begin{array}{l}
c_{3 x} \\
c_{3 y}
\end{array}\right]=\left[\begin{array}{l}
x+d C_{\theta} \\
y+d S_{\theta}
\end{array}\right] .
$$

Hence, the inverse kinematic solution of the mechanism at end-effector pose of $(x, y, \theta)^{T}=(0.5,0.4, \pi / 6)^{T}$ is

$$
\begin{aligned}
\alpha_{1} & =18.22^{\circ} \\
\alpha_{2} & =60.72^{\circ} \\
\alpha_{3} & =210^{\circ} \\
\beta_{1} & =48.19^{\circ} \\
\beta_{2} & =109.47^{\circ} \\
\beta_{3} & =70^{\circ} .
\end{aligned}
$$

To perform the direct kinematic process, it is assumed that only the link lengths and the actuated joint displacement $\alpha_{i}$ $(i=1,2,3)$ are given. Then (18)-(27) are utilised to calculate $(x, y)$ while (29)-(33) are utilised to obtain $\theta$. The results are tabulated in Table I.

It is shown (Table I) that in this example, the solution to the direct kinematic problem has admitted only two real solutions out of the possible four. The two configurations of the real solutions are shown in Fig. 6 . 
TABLE I

THE DIRECT KINEMATIC SOLUTIONS TO THE EXAMPLE 3RRR PLANAR PARALLEL MECHANISM IN SECTION V, SHOWING REAL AND IMAGINARY ROOTS.

\begin{tabular}{|c|c|c|c|}
\hline $\mathrm{x}$ & $\mathrm{y}$ & $\mathrm{T}$ & $\theta$ \\
\hline 0.5 & 0.4 & 0.26795 & $30^{\circ}$ \\
0.5 & 0.4 & 1.19175 & $100^{\circ}$ \\
0.67556 & 0.07397 & $0.75205+0.60969 \mathrm{i}$ & - \\
0.67556 & 0.07397 & $0.75205+0.60969 \mathrm{i}$ & - \\
\hline
\end{tabular}

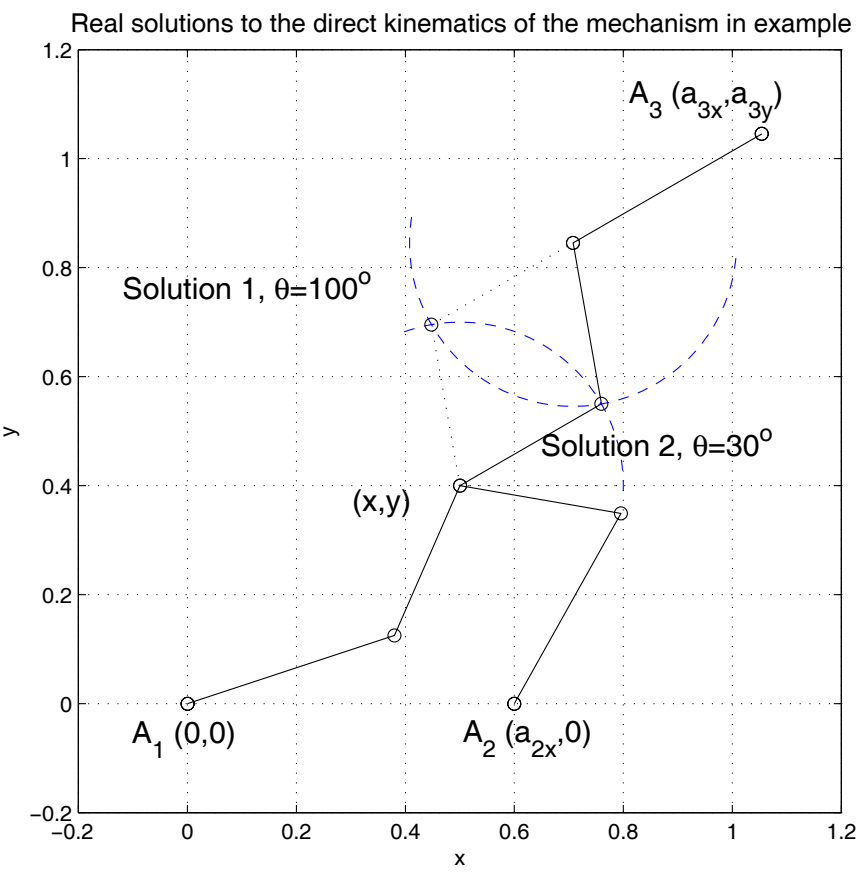

Fig. 6. Configuration of the simplified 3RRR planar parallel mechanism in the example, showing both the real solutions.

\section{CONCLUSION}

In this paper, the direct kinematic problem of a planar parallel mechanism is investigated. Specifically, it focused on obtaining the closed-form solution to the direct kinematics of 3RRR planar parallel mechanisms. A solution to the general 3RRR planar parallel mechanism was presented and shown to be in the form of $8^{\text {th }}$ degree univariate polynomial. For an analytic expression to be obtained, it is necessary to reduce the order of the polynomial solution to 4 or lower. Leveraging from the more established study of 3RPR planar parallel mechanisms where 3 types of simplification resulting in analytic expression had been identified, this paper pursued a simplification similar in idea to one of those established for 3RPR mechanisms. The derivation decouples the solution into two quadratic equations, hence it is very efficient in its computation. A numerical example and verification was presented. This result is very useful in the control strategies of a 3RRR planar parallel manipulator as it is more accurate by obtaining an exact solution and more efficient as it would not require iterative numerical computation to solve for the direct kinematics.

\section{ACKNOWLEDGMENT}

This work is supported by Australian Research Council LIEF grant and Discovery grant.

\section{REFERENCES}

[1] K. Liu, J. M. Fitzgerald, and F. L. Lewis, "Kinematic analysis of a stewart platform manipulator," IEEE Transactions on Industrial Electronics, vol. 40, no. 2, pp. 282-293, Apr 1993.

[2] L.-C. Wang and K.-T. Oen, "Numerical direct kinematic analysis of fully parallel linearly actuated platform type manipulators," Journal of Robotic Systems, vol. 19, no. 8, pp. 391-400, Aug 2002.

[3] R. Boudreau, "Real time solution to the forward kinematic problem of a general spherical three-degree-of-freedom parallel manipulator," in Proceedings of ASME Design Engineering Technical Conferences, vol. 82, no. 1, Boston, MA, USA, 17-20 Sep 1995 1995, pp. 965-971.

[4] O. Didrit, M. Petitot, and E. Walter, "Guaranteed solution of direct kinematic problems for general configurations of parallel manipulators," IEEE Transactions on Robotics and Automation, vol. 14, no. 2, pp. 259 - 266, Apr 1998.

[5] P. Dietmaier, "The Stewart - Gough platform of general geometry can have 40 real postures," in Advances in Robot Kinematics: Analysis and Control, Strobl-Salzburg, Austria, 29 June - 3 July, pp. 7-16.

[6] C. Gosselin and J. Merlet, "Direct kinematics of planar parallel manipulators: special architectures and number of solutions," Mechanism and Machine Theory, vol. 29, no. 8, pp. 1083-1097, 1994.

[7] P. Ji and H. Wu, "Algebraic solution to forward kinematics of a 3 dof spherical parallel manipulator," Journal of Robotic Systems, vol. 18, no. 5, pp. 251-257, May 2001.

[8] I. Bonev, J. Ryu, S.-G. Kim, and S.-K. Lee, "A closed-form solution to the direct kinematics of nearly general parallel manipulators with optimally located three linear extra sensors," IEEE Transactions on Robotics and Automation, vol. 17, no. 2, pp. 148-156, Apr 2001.

[9] L. Baron and J. Angeles, "Direct kinematics of parallel manipulators under joint-sensor redundancy," IEEE Transactions on Robotics and Automation, vol. 16, no. 1, pp. 12-19, Feb 2000.

[10] X. Kong and C. Gosselin, "Forward displacement analysis of third-class analytic 3-rpr planar parallel manipulators," Mechanism and Machine Theory, vol. 36, pp. 1009-1018, 2001.

[11] S. Sreenivasan, K. Waldron, and P. Nanua, "Closed-form direct displacement analysis of a 6-6 stewart platform," Mechanism and Machine Theory, vol. 29, no. 6, pp. 855-864, 1994.

[12] J. Merlet, "Direct kinematics of planar parallel manipulators," Proceedings of IEEE International Conference on Robotics and Automation, pp. 3744 - 3749, Apr 1996.

[13] K. Hunt, "Structural kinematics of in-parallel-actuated robot arms," ASME Journal of Mechanisms, Transmissions and Automation in Design, vol. 105, no. 4, pp. 705-712, 1983.

[14] P. Ji and H. Wu, "An efficient approach to the forward kinematics of a planar parallel manipulator with similar platforms," IEEE Transactions on Robotics and Automation, vol. 18, no. 4, pp. 647-649, 2002.

[15] I. Bonev, D. Zlatanov, and C. Gosselin, "Singularity analysis of 3dof planar parallel mechanisms via screw theory," ASME Journal of Mechanical Design, vol. 125, pp. 573 - 581, 2003.

[16] G. Alici, "An inverse position analysis of five-bar planar parallel manipulators," Robotica, vol. 20, no. 2, pp. 195-201, Mar/Apr 2002.

[17] G. Alici and B. Shirinzadeh, "Optimum synthesis of planar parallel manipulators based on kinematic isotropy and force balancing," Robotica, vol. 22, no. 1, pp. 97-108, Jan/Feb 2004. 